\title{
(S)-(-)-N-[2-(3-Hydroxy-2-oxo-2,3-dihydro-1H-indol-3-yl)-ethyl]-acetamide inhibits colon cancer growth via the STAT1 pathway
}

\author{
Kang $\mathrm{LI}^{1}$, Dawei YUAN ${ }^{1}$, Wei $\mathrm{CHEN}^{1}$, Rulan $\mathrm{MA}^{1}$, Yinsheng XIAN ${ }^{1 *}$ (D)
}

\begin{abstract}
(S)-(-)-N-[2-(3-Hydroxy-2-oxo-2,3-dihydro-1H-indol-3-yl)-ethyl]-acetamide (EA), a new compound isolated from the plant Selaginella pulvinata, has been identified to possess anti-proliferative effects against SK-mel-110 cells in vitro via upregulating the expression of inhibitor of growth family member 4 . The present study aimed to investigate whether EA could exert antitumor effects against colon cancer. The results revealed that EA exerted anti-proliferative effects against a broad spectrum of different types of tumor and could induce apoptosis of colon cancer cells via the signal transducer and activator of transcription 1 pathway. It was demonstrated that treating tumor-bearing mice with EA significantly inhibited colon tumor growth in a xenograft tumor model and syngeneic tumor model. Furthermore, EA treatment reshaped the tumor microenvironment by decreasing myeloidderived suppressor cell accumulation and increasing the tumor infiltrating lymphocytes. The results from the present study suggest that EA could be developed as a potential antitumor drug against colon cancer.
\end{abstract}

Keywords: (S)-(-)-N-[2-(3-Hydroxy-2-oxo-2,3-dihydro-1H-indol-3-yl)-ethyl]-acetamide; colon carcinoma; HT-29; CT26.

Practical Application: EA could be developed as a potential antitumor drug against colon cancer.

\section{Introduction}

As the second most prevalent cause of cancer-associated mortality in women and men after lung cancer, colon cancer arises due to the conversion of precancerous benign polyps present in the inner lining of the colon, which is responsible for nearly 700,000 deaths and 1.4 million new cases worldwide every year (Jaganathan et al., 2014). Despite the steady decline in incidence rates, and the significant increase in survival rates, potentially due to the increase in cancer screening and improved therapy options, a number of problems remain in the clinical setting, including chemoresistance and severe side effects (Desoize et al., 1998; Hazlehurst et al., 2003). Therefore, it is imperative to investigate and identify novel and safer therapeutic agents with new mechanisms of action against colon carcinoma.

Belonging to the signal transducer and activator of transcription (STAT) family, STAT1 prevents the development and progression of established tumors by acting as a tumor suppressor, which could inhibit the neoplastic cell growth via regulation of Bcl-2 and the caspase family (Kim \& Lee, 2007; Chen et al., 2017; Wang \& Koromilas, 2015). Previous studies have demonstrated that STAT1 inhibits the progression of human hepatocellular carcinoma as a negative regulator through inducing apoptosis and cell cycle arrest. Upregulation of STAT1 exerts tumor-suppressive activity in colon cancer cells (Elahi et al., 2008; Chen et al., 2015).

Natural products isolated from plants have gained attention due to their potent biological and pharmaceutical activities. A novel compound termed (S)-(-)-N-[2-(3-Hydroxy-2-oxo-2,3-dihydro$1 H$-indol-3-yl)-ethyl]-acetamide (EA), isolated from the plant Selaginella pulvinata, was observed to inhibit the proliferation of melanoma cells through inducing apoptosis (Wang et al., 2016). In the present study, EA was further analyzed in order to investigate its effects on colon carcinoma. The results revealed that EA exhibited potent antitumor activities, as evidenced by inhibiting tumor growth of colon carcinoma in xenograft tumor model and murine syngeneic tumor model. These results provided new insights to develop EA as a new potential therapeutic agent against colon cancer.

\section{Materials and methods}

\subsection{Cell lines, reagents and chemicals}

Normal human colon mucosal epithelial cell line NCM460, hepatocellular cell line Hep3B, pancreatic adenocarcinoma cell line PANC-1, melanoma cell line B16F10, colorectal adenocarcinoma cell lines HT-29 and SW480, colorectal carcinoma cell line HCT116, murine colon carcinoma cell line CT26, lung adenocarcinoma cell line A549, osteosarcoma cell line MG-63, glioblastoma cell line U251 (catalog no. TCHu 58), and breast adenocarcinoma cell line MCF-7 were purchased from the Shanghai Cell Bank. RPMI1640, DMEM and fetal bovine serum were purchased from Gibco; Thermo Fisher Scientific, Inc.. MTT and caspase activity assay kits were purchased from 
Sigma-Aldrich; Merck KGaA. 5,5',6,6'-tetrachloro-1,1',3,3'tetraethylbenzimidazolylcarbocyanine iodide (JC-1) was purchased from Molecular Probes; Thermo Fisher Scientific, Inc.. Cell Death Detection ELISA ${ }^{\text {plus }}$ kit was purchased from Roche Applied Science. PCR reagents were purchased from Thermo Fisher Scientific, Inc.. Cytochrome-c immunoassay kit was from R\&D Systems, Inc. Tofacitinib was purchased from Sigma-Aldrich; Merck KGaA. Mitomycin C and Taxol were purchased from Shijiazhuang Pharmaceutical Co. Ltd. (Shijiazhuang, China). EA was kindly provided by Professor Cai from Harbin Medical University. All solvents were purchased from Sinopharm Chemical Reagent Co., Ltd. as analytical grade. Taxol and EA were dissolved in DMSO for the in vitro studies. Mitomycin C was prepared with PBS freshly, and EA was freshly prepared in $0.5 \%$ carboxymethylated cellulose prior to use in the in vivo experiments.

\subsection{Mice}

A total of 6-week old nude mice (Balb/c-nu/nu) were purchased from Vital River Ltd. and housed in sterile, pathogenfree conditions at $25^{\circ} \mathrm{C}$ on a 12 -h light/dark cycle. The mice had free access to food and water during the entire experiment. All animal procedures were approved by the Ethical Committee on Animal Care and Use of The First Affiliated Hospital, Xian Jiaotong University and performed in strict accordance with the regulation of the Use of Animals.

\subsection{Cell culture and treatment}

Cells were cultured in DMEM or RPMI1640 medium supplemented with $10 \%$ fetal bovine serum, $100 \mu \mathrm{g} / \mathrm{ml}$ Penicillin/ Streptomycin at $37^{\circ} \mathrm{C}$ in an atmosphere of $5 \% \mathrm{CO}_{2}$. Cells were treated with Taxol or EA for $48 \mathrm{~h}$. Cells treated with DMSO were used as the negative control. The cell viability was measured using an MTT assay. For the mechanism study, HT-29 cells were treated with 1, 3 or $10 \mathrm{nM} \mathrm{EA}$ for $48 \mathrm{~h}$. Cells treated with DMSO were used as the negative control. The concentrations of EA were based on the half maximal inhibitory concentration $\left(\mathrm{IC}_{50}\right)$ value and the preliminary study.

\subsection{Mitochondrial Membrane Potential (MMP) measurement}

MMP was measured using the fluorescent probe JC-1. Briefly, following treatment, HT-29 cells were incubated with JC-1 for $15 \mathrm{~min}$ at $37^{\circ} \mathrm{C}$ in the dark. After rinsing twice, the red/ green fluorescence intensity was determined by the fluorescence microplate reader (TECAN Polarion) at an excitation of $490 \mathrm{~nm}$ and emission of 530/590 $\mathrm{nm}$.

\subsection{Cytochrome-c assay}

HT-29 cells were fractionated following treatment. The cytochrome-c was measured using the assay kit, according to the manufacturer's protocol.

\subsection{DNA fragmentation measurement}

Following treatment, HT-29 cells were lyzed and DNA fragmentation was measured using the Cell Death Detection ELISA ${ }^{\text {plus }}$ kit, according to the manufacturer's protocol. The absorbance was measured using a microplate reader at $405 \mathrm{~nm}$.

\subsection{Caspase activity measurement}

Following treatment, HT-29 cells were lyzed and activities of caspases were determined with the fluorescent assay kit (R\&D Systems, Inc.), according to the manufacturer's protocol.

\subsection{Apoptosis analysis}

Following treatment, $3 \times 10^{6}$ HT- 29 cells were collected and washed with pre-chilled PBS. Cells were then re-suspended in $500 \mu \mathrm{L}$ binding buffer and incubated with $5 \mu \mathrm{L}$ Annexin V-FITC/ propidium iodide for $10 \mathrm{~min}$ in the dark at room temperature. Apoptosis was detected using a FACScan flow cytometer (Becton, Dickinson and Company). Data were analyzed using FlowJo software (version 10.0; FlowJo LLC).

\subsection{Reverse transcription-quantitative PCR}

Total RNA was extracted with the Qiagen RNeasy reagent according to the manufacturer's protocol and mRNA was reverse transcribed into cDNA using SuperScript master mix (Bio-Rad Laboratories, Inc.) Quantitative PCR was performed using a StepOne system, SYBR green Supermix (both from Thermo Fisher Scientific, Inc.) and the $2^{-\Delta \Delta C_{q}}$ method. The gene expression was normalized to $\beta$-actin. The primer sequences were as follows: Caspase- 3 forward, TGGAATTGATGCGTGATGTT and reverse, GGCAGGCCTGAATAATGAAA; caspase- 9 forward, CTAGTTTGCCCACACCCAGT and reverse, GCATTAGCGACCCTAAGCAG; melanoma-associated antigen (MAGE-A) 1 forward, ATGGCTGACTCCCGTAACAC and reverse, TCCTCAGATGGGCTATCAGG; MAGE-A3 forward, CTCAGGCCCAACAGGAATTA and reverse, AGGCCCTCTGATCCTTTGAT; Homo sapiens $\beta$-actin forward, CGTACACCTCTGGCCGTACT and reverse, AGGGCAACATAGCACAGCTT; and Mus musculus $\beta$-actin forward, AGCCATGTACGTAGCCATCC and reverse, GCTGTGGTGGTGAAGCTGTA.

\subsection{Western blot analysis}

Following treatment, cells were lysed using RIPA buffer (Sigma Aldrich; Merck KGaA), supplemented with protease inhibitors (Roche Diagnostics), phosphatase inhibitors (Thermo Fisher Scientific, Inc.), $1 \mathrm{mM}$ EDTA and $1 \mathrm{mM}$ PMSF (Sigma Aldrich; Merck KGaA). Protein concentration was determined with the protein assay kit (Beyotime Institute of Biotechnology). After boiling for $5 \mathrm{~min}, 40 \mu \mathrm{g}$ sample protein with $4 \mathrm{x}$ loading dye per lane was separated via SDS-PAGE (4-12\% gel). The proteins were then transferred to polyvinylidene difluoride membranes. After blocking with $1 \%$ bovine serum albumin for $1 \mathrm{~h}$, membranes were incubated with different primary 
antibodies, including anti-cleaved Caspase- 3 rabbit polyclonal antibody (pAb; 1:500; catalog no. ab49822); anti-cleaved Caspase-9 rabbit pAb (1:500; catalog no. ab2324); anti-Bax rabbit pAb (1:1,000; catalog no. ab199677); anti-Bcl-2 rabbit pAb (1:1,000; catalog no. ab196495); anti-STAT1 (phospho Y701) rabbit pAb (1:1,000; catalog no. ab30645); anti-STAT1 rabbit monoclonal antibody (mAb) (1:5,000; catalog no. ab109320); anti-STAT2 (phospho Y690) rabbit pAb (1:500; catalog no. ab53132); anti-STAT2 rabbit mAb (1:5,000; catalog no. ab32367); anti-STAT3 (phospho Y705) rabbit mAb (1:3,000; catalog no. ab76315); anti-STAT3 rabbit pAb (1:1,500; catalog no. ab226942); anti- $\beta$-actin rabbit pAb (1:3,000; catalog no. ab8227) overnight at $4{ }^{\circ} \mathrm{C}$, and then incubated with the corresponding secondary horseradish peroxidase goat anti-rabbit IgG H\&L antibody (1:5,000; catalog no. ab205718) for $1 \mathrm{~h}$. The bands were detected using an enhanced chemiluminescence system.

\subsection{Flow cytometry analysis}

Tumors were cut into small pieces and digested using a tumor disassociation kit (Miltenyi Biotec, Inc.), followed by filtering with $70-\mu \mathrm{m}$ cell strainers. Mononuclear cells were then enriched by percoll gradient centrifugation, followed by staining with anti-CD11b APC-cy7, anti-Ly6G APC, anti-Ly6C AF700, anti-CD4 Percp, anti-CD8 FITC, anti-IFN $\gamma$ PE-CF594, and anti-granzyme B PE antibodies (all from BD Biosciences) for flow cytometry analysis using a BD FACSCalibur.

\subsection{Xenograft tumor model}

A total of $5 \times 10^{6}$ HT-29 cells were injected subcutaneously into the right flank of the nude mice. When the tumor volume reached $50-100 \mathrm{~mm}^{3}$, tumor-bearing mice were divided into four groups (5 mice per group), and treatment was administered. Mitomycin C (5 mg/kg) was injected intraperitoneally twice every week. EA ( 5 and $10 \mathrm{mg} / \mathrm{kg}$ ) was given orally 6 days per week, and mice treated with PBS were used as negative controls. The in vivo dosage was based on the toxicity, pharmacokinetics and pharmacodynamics of the compound. The dosage of EA in the animal model was based on the preliminary study, which revealed that administration of EA at 5 and $10 \mathrm{mg} / \mathrm{kg}$ did not cause toxicity to mice, but demonstrated antitumor effects (data not shown). When the tumor volume reached $1,500 \mathrm{~mm}^{3}$ (at $\sim 3.5$ weeks), all tumor-bearing mice were sacrificed via initial inhalation of $\mathrm{CO}_{2}$ at a flow rate of $1.6 \mathrm{l} / \mathrm{min}$ in the $8 \mathrm{l}$ chamber for $5 \mathrm{~min}$ and then via cervical dislocation to collect the tumors for analysis.

\subsection{Syngeneic tumor model}

A total of $5 \times 10^{5} \mathrm{CT} 26$ cells were injected into Balb/cJ mice subcutaneously. When the tumor volume reached $50-100 \mathrm{~mm}^{3}$, tumor-bearing mice were divided into three groups ( 5 mice per group), and treatment was administered. EA ( 5 and $10 \mathrm{mg} / \mathrm{kg}$ ) was given orally 6 days per week, and mice treated with PBS were used as negative controls. When the tumor volume reached $1,500 \mathrm{~mm}^{3}$ (at $\sim 4$ weeks), all tumor-bearing mice were sacrificed via initial inhalation of $\mathrm{CO}_{2}$ at a flow rate of $1.6 \mathrm{l} / \mathrm{min}$ in the $8 \mathrm{l}$ chamber for $5 \mathrm{~min}$ and then via cervical dislocation to collect the tumors for analysis.

\subsection{Statistical analysis}

Data are expressed as the mean \pm standard deviation, and SPSS software (version 12.0; SPSS, Inc.) was used for the statistical analysis. Data were analyzed by one-way analysis of variance followed by Fisher's least significant difference test. $\mathrm{P}<0.05$ was considered to indicate a statistically significant difference.

\section{Results}

\subsection{EA inhibits cancer cell proliferation}

A MTT assay was used to determine the anti-proliferative activities of EA on different cancer cell lines. EA selectively inhibited the proliferation of colon cancer and melanoma cells. Furthermore, EA exhibited low toxicity to the normal human colon mucosal epithelial cell line NCM460 (Table 1).

Table 1. Growth inhibition of cultured cancer cells by EA.

\begin{tabular}{cccc}
\hline Cell line & Origin & EA IC $_{50} \mathrm{nM}$ & Taxol IC $_{50}, \mathrm{nM}^{\circ}$ \\
\hline NCM460 & Normal human colon mucosal epithelial cell & $>5,000$ & $>5,000$ \\
Hep3B & Hepatocellular & $1,253.3 \pm 113.5$ & $38.5 \pm 3.5$ \\
PANC-1 & Pancreatic adenocarcinoma & $162.3 \pm 15.5$ & $16.7 \pm 1.9$ \\
B16F10 & Melanoma & $8.35 \pm 2.1$ & $19.5 \pm 1.8$ \\
HT-29 & Colorectal adenocarcinoma & $9.6 \pm 2.8$ & $13.7 \pm 3.1$ \\
SW-480 & Colorectal adenocarcinoma & $11.5 \pm 3.2$ & $10.6 \pm 2.8$ \\
HCT 116 & Colorectal carcinoma & $15.8 \pm 4.6$ & $13.9 \pm 3.7$ \\
CT26 & Colon carcinoma & $8.62 \pm 2.3$ & $9.53 \pm 2.6$ \\
A549 & Lung adenocarcinoma & $44.5 \pm 5.5$ & $9.3 \pm 1.9$ \\
MG-63 & Osteosarcoma & $112.7 \pm 13.2$ & $14.1 \pm 2.7$ \\
U251 & Glioblastoma & $1,357.6 \pm 125.8$ & $13.3 \pm 2.5$ \\
MCF-7 & Breast adenocarcinoma & $1,765.7 \pm 165.1$ & $6.5 \pm 1.3$ \\
\hline
\end{tabular}

EA, (5)-(-)-N-[2-(3-Hydroxy-2-oxo-2,3-dihydro-1H-indol-3-yl)-ethyl] acetamide; $\mathrm{IC}_{50}$, half maximal inhibitory concentration. 


\subsection{EA induces apoptosis in HT-29 cells}

Following EA treatment, the apoptosis-associated biomarkers were detected. When compared with the control group, EA treatment decreased the MMP (Figure 1A), increased cytochrome-c release (Figure 1B), increased DNA fragmentation (Figure 1C), increased caspase-3/9 activities in HT-29 cells (Figure 1D), and increased the percentage of apoptotic cells (Figure 1E), all in a dose-dependent manner. Expression levels of pro-apoptotic genes and proteins increased, but anti-apoptotic protein expression significantly decreased following EA treatment (Figure 2A, B).
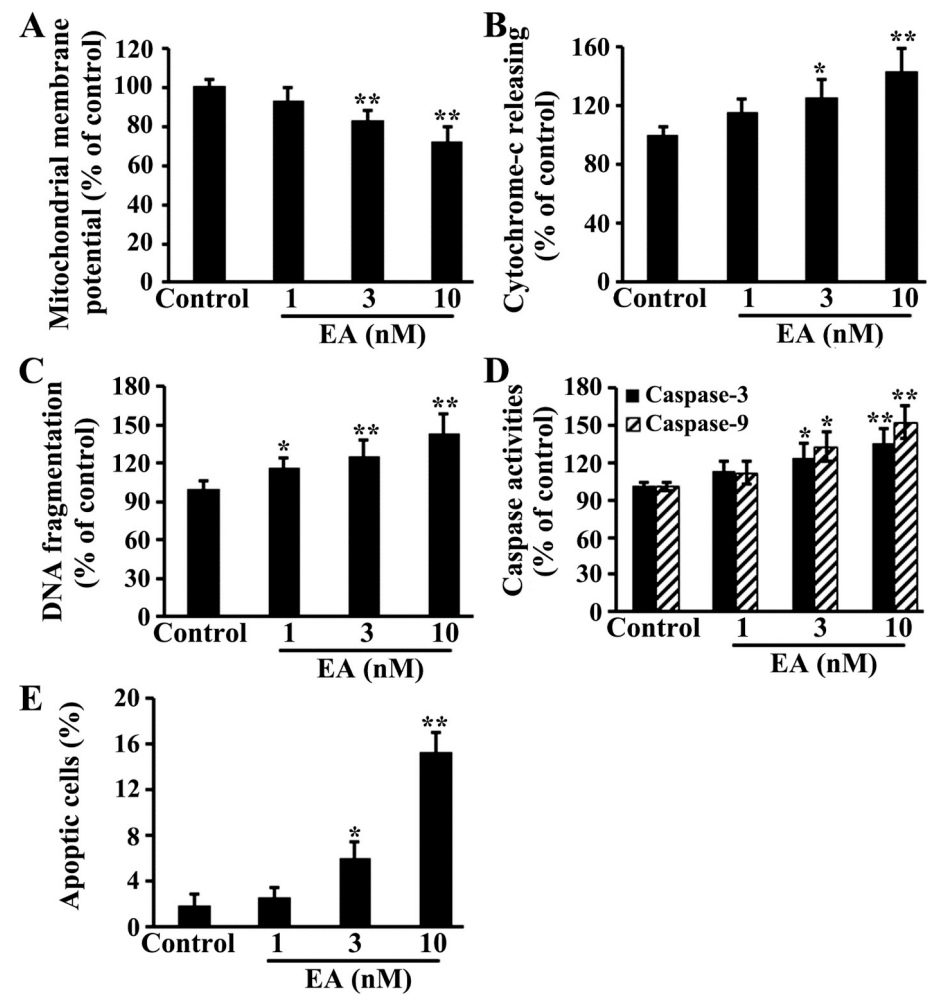

Figure 1. EA induces apoptosis in HT-29 cells. HT-29 cells were treated with EA for $48 \mathrm{~h}$ followed by detection of apoptosis-associated markers. Cells treated with DMSO were used as the negative control. (A) EA treatment decreased mitochondrial membrane potential; (B) increased the cytochrome-c release; (C) increased DNA fragmentation; (D) increased caspase activities; and (E) increased the amount of apoptotic cells detected by Annexin V/propidium iodide staining. Data are expressed as the mean \pm standard deviation $(\mathrm{n}=3)$. ${ }^{*} \mathrm{P}<0.05$, ${ }^{* *} \mathrm{P}<0.01$ vs. control group. EA, (5)-(-)-N-[2-(3-Hydroxy-2-oxo-2,3-dihydro-1H-indol-3-yl)-ethyl]acetamide.
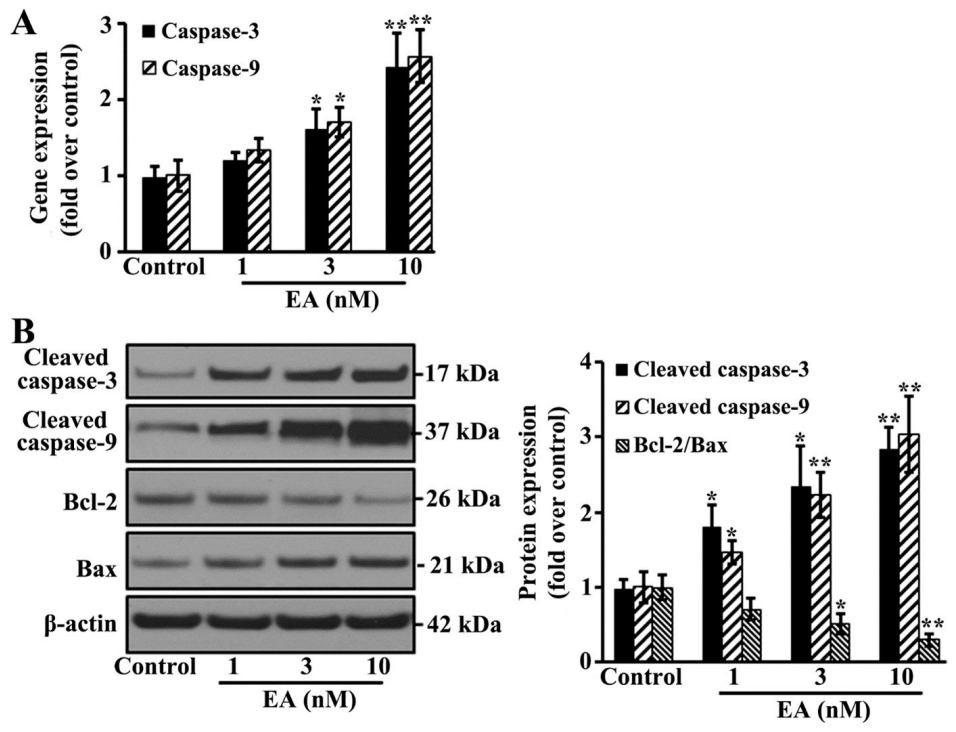

Figure 2. EA treatment regulates apoptosis-associated gene and protein expression. (A) EA increased caspase gene expression; (B) Effects of EA on apoptosis-associated protein expression; Data are expressed as the mean \pm standard deviation $(\mathrm{n}=3)$. ${ }^{*} \mathrm{P}<0.05,{ }^{* *} \mathrm{P}<0.01$ vs. control group. EA, (5)-(-)-N-[2-(3-Hydroxy-2-oxo-2,3-dihydro-1H-indol-3-yl)-ethyl]acetamide. 
Furthermore, EA treatment selectively upregulated the expression of p-STAT1, which was repressed by the specific inhibitor of STAT phosphorylation tofacitinib (Figure 3).

\subsection{EA inhibits tumor growth in a xenograft mouse model}

Tumor-bearing mice were treated with 5 or $10 \mathrm{mg} / \mathrm{kg}$ EA. The $10 \mathrm{mg} / \mathrm{kg}$ EA treatment group exhibited significantly inhibited
HT-29 tumor growth (Figure 4A, B), indicated by both tumor volume and tumor weight. Furthermore, EA treatment significantly upregulated the expression of p-STAT1 (Figure 4C, D).

\subsection{EA inhibits tumor growth in the syngeneic mouse model}

The antitumor effects of EA were further investigated in the syngeneic tumor model. Mice-bearing CT26 tumors

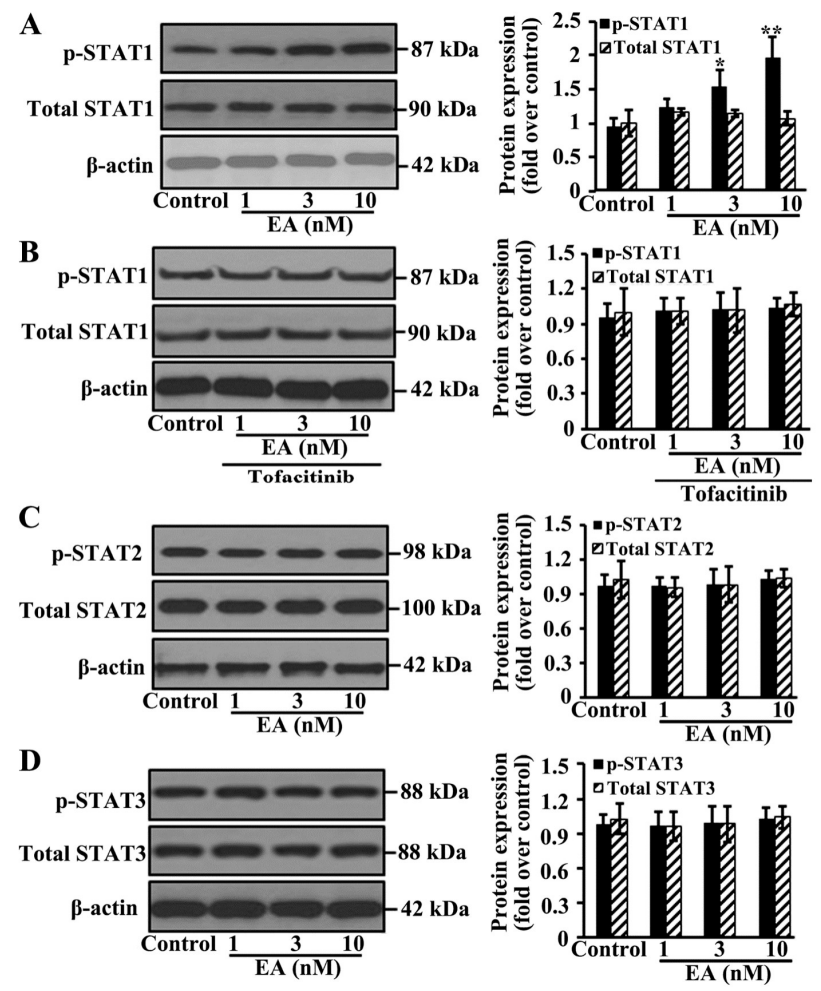

Figure 3. EA selectively increases the protein expression of STAT1. HT-29 cells were treated with EA for $48 \mathrm{~h}$ followed by western blot analysis. (A) EA increased the protein expression of p-STAT1 in HT-29 cells; (B) Tofacitinib repressed the upregulation of p-STAT1 caused by EA; Protein expression of (C) STAT2 and (D) STAT3 was unaffected by EA treatment in HT-29 cells. Data are expressed as the mean \pm standard deviation $(\mathrm{n}=3) .{ }^{*} \mathrm{P}<0.05,{ }^{\text {" }} \mathrm{P}<0.01$ vs. control group. EA, (5)-(-)-N-[2-(3-Hydroxy-2-oxo-2,3-dihydro-1H-indol-3-yl)-ethyl]acetamide; STAT, signal transducer and activator of transcription; $\mathrm{p}$, phosphorylated.

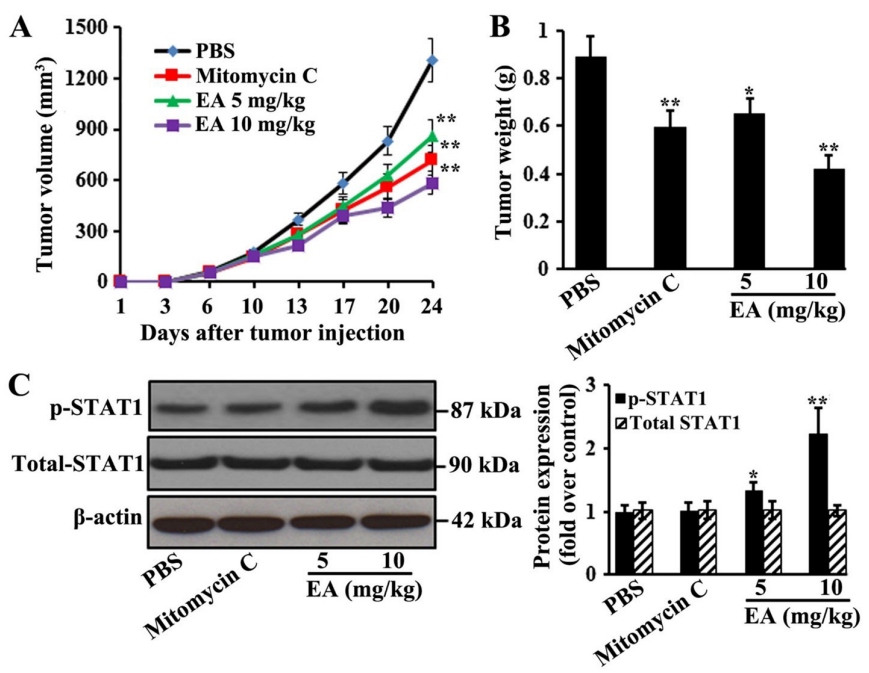

Figure 4. EA treatment inhibits the HT-29 tumor growth in nude mice. (A) Tumor volume was measured twice per week; (B) Tumor weight was recorded at the end of experiment; (C) STAT1 protein expression was measured in tumors. ${ }^{*} \mathrm{P}<0.05,{ }^{* *} \mathrm{P}<0.01 \mathrm{vs}$. PBS control. EA, (5)-(-)-N-[2(3-Hydroxy-2-oxo-2,3-dihydro-1H-indol-3-yl)-ethyl]acetamide; STAT, signal transducer and activator of transcription; p, phosphorylated. 
were sacrificed to collect tumors for analysis when the tumor volume reached $1,500 \mathrm{~mm}^{3}$ in the negative control group. EA treatment significant inhibited tumor growth and decreased the tumor burden of tumor-bearing mice (Figure 5A, B). Furthermore, EA treatment increased tumor-infiltrating lymphocytes and decreased myeloid derived suppressive cells. EA treatment also increased the neo-antigen expression (Zajac et al., 2017) and production of IFN $\gamma$, as well as the production of granzyme B in tumor tissues (Figure 6).
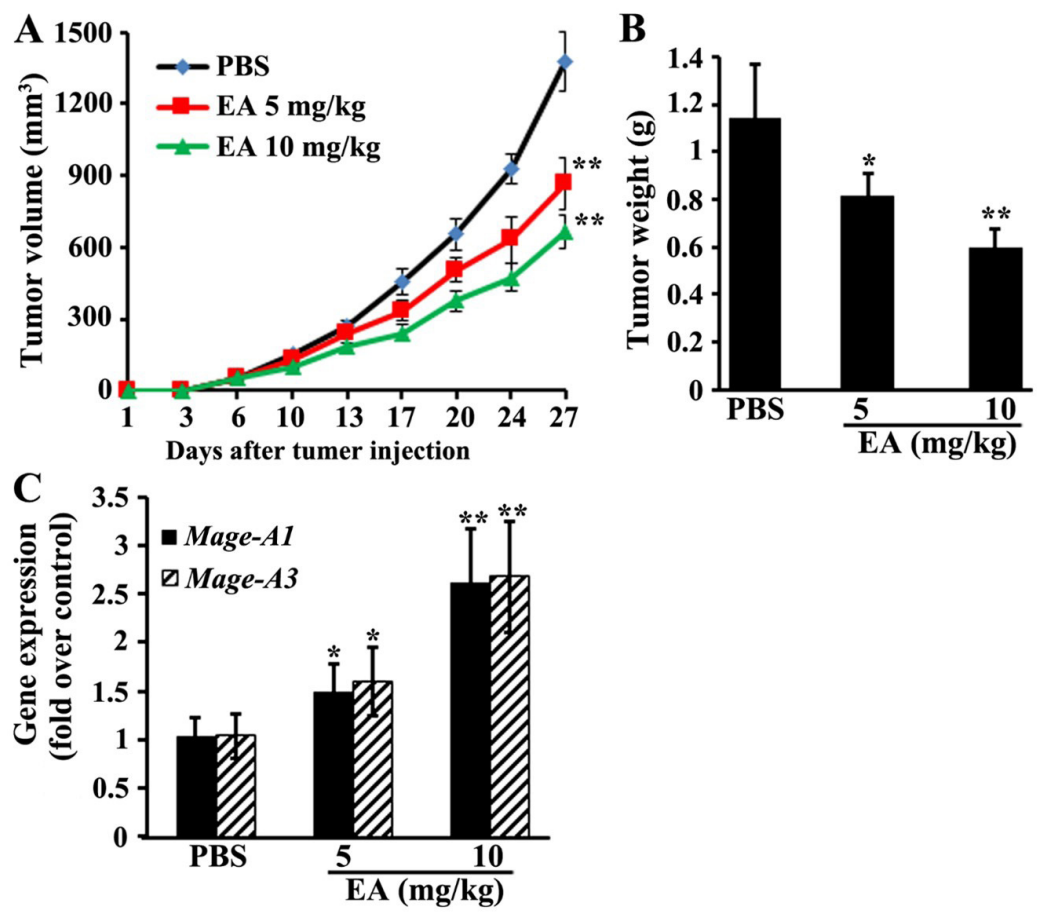

Figure 5. EA treatment inhibits the growth of CT26 tumors in the syngeneic tumor model. CT26 tumor-bearing mice were treated with EA or PBS. (A and B) EA treatment delayed the tumor growth; and (C) increased the expression of specific genes MAGE-A1 and MAGE-A3. Data are expressed as the mean \pm standard deviation $(\mathrm{n}=3)$. ${ }^{*} \mathrm{P}<0.05,{ }^{* *} \mathrm{P}<0.01$ vs. PBS control. EA, (5)-(-)-N-[2-(3-Hydroxy-2-oxo-2,3-dihydro-1Hindol-3-yl)-ethyl]acetamide; MAGE-A, melanoma-associated antigen.
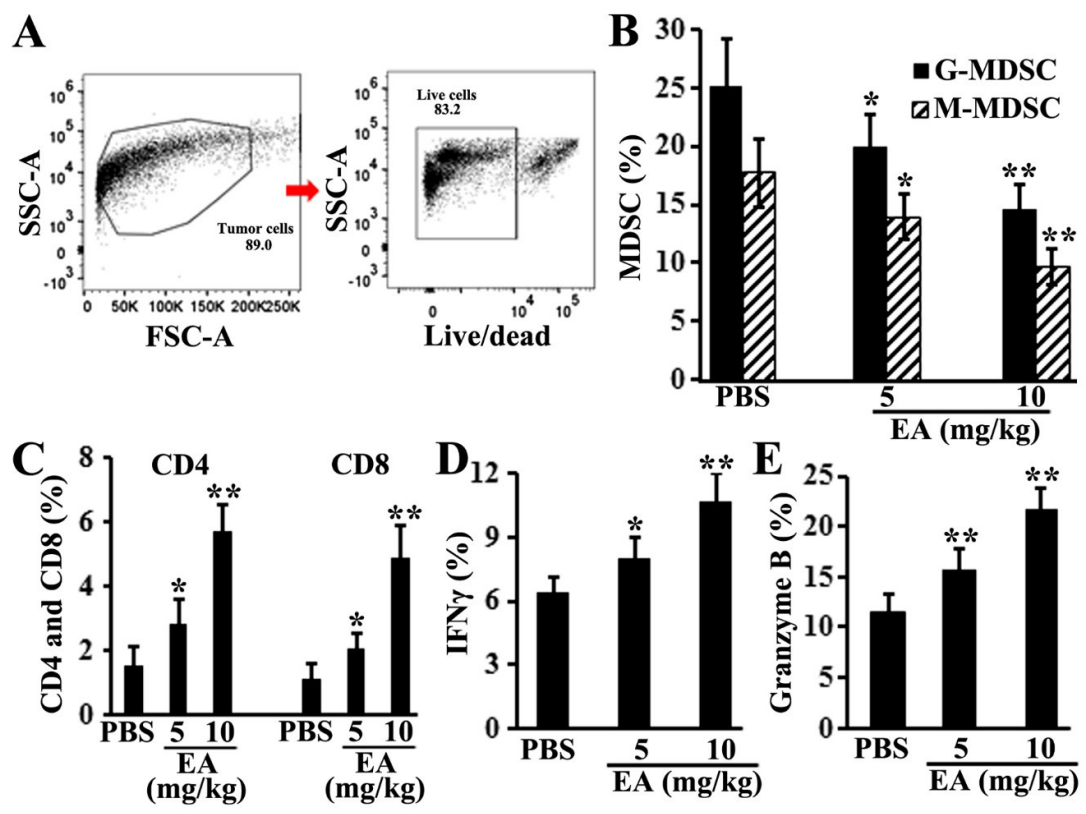

Figure 6. EA treatment primes the tumor microenvironment. (A) Representative flow cytometry graphics complete with the gating strategy; (B) EA decreased the accumulation of MDSC in tumors; (C) increased tumor-infiltrating lymphocytes; and (D) increased the production of IFN $\gamma$; and (E) granzyme B in tumors. Data are expressed as the mean \pm standard deviation $(\mathrm{n}=3) .{ }^{*} \mathrm{P}<0.05,{ }^{* \star} \mathrm{P}<0.01$ vs. PBS control. EA, (5)-(-)-N-[2(3-Hydroxy-2-oxo-2,3-dihydro-1H-indol-3-yl)-ethyl]acetamide; MDSC, myeloid-derived suppressor cells. 


\section{Discussion}

Despite progress towards the outcomes of patients with colorectal cancer over the last decade, with the overall survival increasing from 14.2 months with 5-fluorouracil-based monotherapy to near 30.0 months with targeted agent combinations with FOLFOX- or FOLFIRI-based chemotherapy, the 5-year survival rates remain at $<15 \%$ for patients with stage IV disease (Cummings et al., 2007). Recently, research has shifted towards the oral drug delivery instead of injection, as administering drugs via the oral route exhibits maximum absorption of drugs, improves patient quality of life and is cost-effective (Butt et al., 2018). In the present study, it was reported that EA, a natural compound extracted from the leaves of Selaginella pulvinata, activated caspase activities, leading to apoptosis of colon cancer cells through upregulating STAT1 expression. EA selectively inhibited the proliferation of colon cancer and melanoma cells, as demonstrated by the $\mathrm{IC}_{50}$ values. An $\mathrm{IC}_{50}$ value is a good marker to observe the inhibitory effects of EA, and is also an easy method of comparing the efficacy between different cell lines or different antitumor agents. This assay was used in the present study to screen the compound against $>10$ cell lines and further investigate the underlying molecular mechanism when used against colon cancer. Other assays, such as cell counts and cell cycle analyses, will form the focus of future studies.

Mitochondria are important during the process of cell death regulation, and the decrease in the MMP would induce the cytochrome-c release from the mitochondria to the nucleus (Chiang et al., 2011), resulting in the activation of caspase-associated apoptotic proteins to facilitate the formation of apoptosome complex (Xu et al., 2009). Furthermore, the cytochrome-c release also activates caspase-9. Acting as an apoptotic executor, caspase- 3 activates DNA fragmentation factor, which in turn activates endonucleases to cleave nuclear DNA, and ultimately leads to cell death (Liu et al., 2015). As a biochemical hallmark, DNA double stranded breaks have been widely used as an apoptosis index (Kuo \& Yang, 2008; May \& Madge, 2007). The results of the present study demonstrated that DNA fragmentation significantly increased following treatment with EA. Furthermore, treatment with EA also decreased the MMP, increased cytochrome-c release and increased the activities of caspase-3/caspase-9. Anti-apoptotic protein Bcl-2 expression decreased, but pro-apoptotic protein Bax, as well as caspase, expression increased.

The upregulation of STAT1 in the present study following EA treatment was notable, as STAT1 is a known tumor suppressor and regulator of the pro-apoptotic responses (Abroun et al., 2015; Koromilas \& Sexl, 2013), and is involved in the inhibition of cell proliferation in colon cancer cells (Souissi et al., 2012). Furthermore, STAT1 enhanced the transcriptional activity of p53 in several p53-responsive pro-apoptotic genes and to negatively regulate the Bcl-xL promoter (Meng et al., 2017). The results from the present study suggested that STAT1 could be involved in the anti-proliferative and pro-apoptotic effects of EA in colon cancer. However, the exact molecular mechanisms underlying this regulation require further investigation.

Previous studies have demonstrated that STAT1 could be upregulated by IFN- $\gamma$ (Fulda \& Debatin, 2002). The results of these studies revealed that the inactivation of the STAT pathway was associated with the acquired resistance of PD-1 blockade therapy in melanoma (Yaguchi \& Kawakami, 2016; Shin et al., 2017). IFN- $\gamma$ are currently used in combination with targeted anticancer agents, conventional chemotherapeutics and immunostimulatory agents in clinical trials (Zitvogel et al., 2015). Therefore, the activation of STAT1 by EA may be beneficial to enhance the antitumor effects of immunotherapy. The EA-induced activation of STAT1 inhibited tumor growth, leading to the observed potentiation of the IFN- $\gamma$-induced anti-proliferative effects in colon cancer cells.

In the clinical setting, the most frequently used cancer treatments are radiotherapy, chemotherapy and targeted therapy. However, the side effects limit their applications (Schneider et al., 2015; Yock \& Caruso, 2012; Le et al., 2014). Therefore, much more effective treatment is required in order to maximally inhibit tumor development and decrease adverse effects. Immunotherapy has been demonstrated as a promising breakthrough treatment modality against cancer, particularly following the discovery of immune checkpoint inhibitors. It is well-known that the immune system and tumors form an immune equilibrium in order to exist in harmony. Certain tumor cells can be eliminated, but those cells with low immunogenicity survived. Simultaneously, these tumor cells can also change their microenvironment to build an immunosuppressive network by producing immunoregulatory or immunosuppressive cells, including MDSC, tumor-associated macrophages and regulatory T cells (Spranger, 2016; McAllister \& Weinberg, 2014; Lim et al., 2014). Eventually, tumor cells are able to escape from the immune clearance, proliferate and metastasize. In the present study, EA treatment decreased suppressive populations of MDSC and delayed tumor progression. In addition, EA treatment reshaped the tumor microenvironment by increasing the neo-antigen expression and the infiltration of both CD4+ and CD8+ T cells, as well as the production of IFN $\gamma$ and granzyme B. Therefore, the potential application of the present results raises the prospect that clinical studies of EA may be beneficial in combination with checkpoint blockade.

Herbal plants are gaining increasing attention as a source of medicinal products. The present study demonstrated that EA isolated from Selaginella pulvinata inhibited colon tumor growth, providing the scientific rationale to develop EA as a therapeutic agent against colon cancer.

\section{Ethical approval}

All animal procedures were performed under the regulation of the Use of Animals and were approved by the Ethical Committee on Animal Care and Use of The First Affiliated Hospital, Xi'an Jiaotong University.

\section{Conflict of interest}

The author declare that they have no conflict of interest.

\section{Availability of data and material}

The datasets used or analysed during the current study are available from the corresponding author on reasonable request.

\section{Funding}

The authors declare that no financial support was received 


\section{References}

Abroun, S., Saki, N., Ahmadvand, M., Asghari, F., Salari, F., \& Rahim, F. (2015). STATs: an old story, yet mesmerizing. Cell Journal, 17(3), 395-411. PMid:26464811.

Butt, A. S., Nisar, N., \& Mughal, T. (2018). A review: therapeutics potentials of phytochemical drugs and their loading in $\mathrm{pH}$ specific degradable Nano-drug carrier targeting colorectal cancer. JPMA. The Journal of the Pakistan Medical Association, 68(4), 607-614. PMid:29808052.

Chen, J., Wang, H., Wang, J., Huang, S., \& Zhang, W. (2015). STAT1 inhibits human hepatocellular carcinoma cell growth through induction of p53 and Fbxw7. Cancer Cell International, 15(1), 111. http://dx.doi.org/10.1186/s12935-015-0253-6. PMid:26617467.

Chen, Y., Fang, L., Li, G., Zhang, J., Li, C., Ma, M., Guan, C., Bai, F., Lyu, J., \& Meng, Q. H. (2017). Synergistic inhibition of colon cancer growth by the combination of methylglyoxal and silencing of glyoxalase I mediated by the STAT1 pathway. Oncotarget, 8(33), 54838-54857. http://dx.doi.org/10.18632/oncotarget.18601. PMid:28903386.

Chiang, J. H., Yang, J. S., Ma, C. Y., Yang, M. D., Huang, H. Y., Hsia, T. C., Kuo, H. M., Wu, P. P., Lee, T. H., \& Chung, J. G. (2011). Danthron, an anthraquinone derivative, induces DNA damage and caspase cascades-mediated apoptosis in SNU-1 human gasric cancer cells through mitochondrial permeability transition pores and Baxtriggered pathways. Chemical Research in Toxicology, 24(1), 20-29. http://dx.doi.org/10.1021/tx100248s. PMid:21126053.

Cummings, L. C., Payes, J. D., \& Cooper, G. S. (2007). Survival after hepatic resection in metastatic colorectal cancer: a populationbased study. Cancer, 109(4), 718-726. http://dx.doi.org/10.1002/ cncr.22448. PMid:17238180.

Desoize, B., Gimonet, D., \& Jardillier, J. C. (1998). Multicellular resistance: another mechanism for multidrug resistance? Bulletin $d u$ Cancer, 85(9), 785. PMid:9770593.

Elahi, A., Zhang, L., Yeatman, T. J., Gery, S., Sebti, S., \& Shibata, D. (2008). HPP1-mediated tumor suppression requires activation of STAT1 pathways. International Journal of Cancer, 122(7), 1567-1572. http://dx.doi.org/10.1002/ijc.23202. PMid:18059030.

Fulda, S., \& Debatin, K. M. (2002). IFNgamma sensitizes for apoptosis by upregulating caspase- 8 expression through the Stat 1 pathway. Oncogene, 21(15), 2295-2308. http://dx.doi.org/10.1038/sj.onc.1205255. PMid:11948413.

Hazlehurst, L. A., Landowski, T. H., \& Dalton, W. S. (2003). Role of the tumor microenvironment in mediating de novo resistance to drugs and physiological mediators of cell death. Oncogene, 22(47), 73967402. http://dx.doi.org/10.1038/sj.onc.1206943. PMid:14576847.

Jaganathan, S. K., Vellayappan, M. V., Narasimhan, G., Supriyanto, E., Octorina Dewi, D. E., Narayanan, A. L., Balaji, A., Subramanian, A. P., \& Yusof, M. (2014). Chemopreventive effect of apple and berry fruits against colon cancer. World Journal of Gastroenterology, 20(45), 17029-17036. http://dx.doi.org/10.3748/wjg.v20.i45.17029. PMid:25493015.

Kim, H. S., \& Lee, M. S. (2007). STAT1 as a key modulator of cell death. Cellular Signalling, 19(3), 454-465. http://dx.doi.org/10.1016/j. cellsig.2006.09.003. PMid:17085014.

Koromilas, A. E., \& Sexl, V. (2013). The tumor suppressor function of STAT1 in breast cancer. JAK-STAT, 2(2), e23353. http://dx.doi. org/10.4161/jkst.23353. PMid:24058806.

Kuo, L. J., \& Yang, L. X. (2008). Gamma-H2AX-a novel biomarker for DNA double-strand breaks. In Vivo, 22(3), 305-309. PMid:18610740.

Le, D. L., Cao, H., \& Yang, L. X. (2014). Cardiotoxicity of moleculartargeted drug therapy. Anticancer Research, 34(7), 3243-3249. PMid:24982327.

Lim, H. X., Hong, H. J., Cho, D. H., \& Kim, T. S. (2014). IL-18 enhances immunosuppressive responses by promoting differentiation into monocytic myeloid-derived suppressor cells. Journal of Immunology, 193(11), 5453-5460. http://dx.doi.org/10.4049/jimmunol.1401282. PMid:25362180.

Liu, H., Zhao, M., Yang, S., Gong, D. R., Chen, D. Z., \& Du, D. Y. (2015). $(2 \mathrm{R}, 3 \mathrm{~S})$-pinobanksin-3-cinnamate improves cognition and reduces oxidative stress in rats with vascular dementia. Journal of Natural Medicines, 69(3), 358-365. http://dx.doi.org/10.1007/s11418-0150901-0. PMid:25808015.

May, M. J., \& Madge, L. A. (2007). Caspase inhibition sensitizes inhibitor of NF-kappa B kinase beta-deficient fibroblasts to caspaseindependent cell death via the generation of reactive oxygen species. The Journal of Biological Chemistry, 282(22), 16105-16116. http:// dx.doi.org/10.1074/jbc.M611115200. PMid:17430892.

McAllister, S. S., \& Weinberg, R. A. (2014). The tumour-induced systemic environment as a critical regulator of cancer progression and metastasis. Nature Cell Biology, 16, 717-727.

Meng, C., Guo, L. B., Liu, X., Chang, Y. H., \& Lin, Y. (2017). Targeting STAT1 in both cancer and insulin resistance diseases. Current Protein \& Peptide Science, 18(2), 181-188. http://dx.doi.org/10.2174/13892 03718666161117114735. PMid:27855602.

Schneider, B. P., Hershman, D. L., \& Loprinzi, C. (2015). Symptoms: chemotherapy-induced peripheral neuropathy. Advances in Experimental Medicine and Biology, 862, 77-87. http://dx.doi.org/10.1007/978-3319-16366-6_6. PMid:26059930.

Shin, D. S., Zaretsky, J. M., Escuin-Ordinas, H., Garcin-Diaz, A., HuLieskovan, S., Kalbasi, A., Grasso, C. S., Hugo, W. \& Sandoval S. (2017). Primary resistance to PD-1 blockade mediated by JAK1/2 mutations. Cancer Discovery, 7, 188-201.

Souissi, I., Ladam, P., Cognet, J. A., Coquil, S., Varin-Blank, N., BaranMarszak, F., Metelev, V., \& Fagard, R. (2012). A STAT3-inhibitory hairpin decoy oligodeoxynucleotide discriminates between STAT1 and STAT 3 and induces death in a human colon carcinoma cell line. Molecular Cancer, 11(1), 12. http://dx.doi.org/10.1186/1476-459811-12. PMid:22423663.

Spranger, S. (2016) Mechanisms of tumor escape in the context of the T-cell-inflamed and the non-T-cell-inflamed tumor microenvironment. International Immunology, 28(8), 383-391.

Wang, S., \& Koromilas, A. E. (2015). STAT1-mediated translational control in tumor suppression and antitumor therapies. Molecular \& Cellular Oncology, 3(3), e1055049. http://dx.doi.org/10.1080/23 723556.2015.1055049. PMid:27314074.

Wang, Y. D., Zhang, J. Z., Wang, Y. H., Yang, J., Li, H. Y., \& Cai, L. M. (2016). Anti-proliferative constituents from Selaginella pulvinata. Phytochemistry Letters, 15, 26-29. http://dx.doi.org/10.1016/j. phytol.2015.10.021.

Xu, Y. H., Zhao, L. J., \& Li, Y. (2009). Alisol B acetate induces apoptosis of SGC7901 cells via mitochondrial and phosphatidylinositol 3-kinases/ Akt signaling pathways. World Journal of Gastroenterology, 15(23), 2870-2877. http://dx.doi.org/10.3748/wjg.15.2870. PMid:19533808.

Yaguchi, T., \& Kawakami, Y. (2016). Cancer-induced heterogeneous immunosuppressive tumor microenvironments and their personalized modulation. International Immunology, 28(8), 393-399. http://dx.doi. org/10.1093/intimm/dxw030. PMid:27401477.

Yock, T. I., \& Caruso, P. A. (2012). Risk of second cancers after photon and proton radiotherapy: a review of the data. Health Physics, 103(5), 577-585. http://dx.doi.org/10.1097/HP.0b013e3182609ba4. PMid:23032887.

Zajac, P., Schultz-Thater, E., Tornillo, L., Sadowski, C., Trella, E., Mengus, C., Iezzi, G., \& Spagnoli, G. C. (2017). Mage-A antigens and cancer immunotherapy. Frontiers in Medicine, 4, 18. PMid:28337438.

Zitvogel, L., Galluzzi, L., Kepp, O., Smyth, M. J., \& Kroemer, G. (2015). Type I interferons in anticancer immunity. Nature Reviews. Immunology, 15(7), 405-414. http://dx.doi.org/10.1038/nri3845. PMid:26027717. 